Original Paper http://ajol.info/index.php/ijbcs http://indexmedicus.afro.who.int

\title{
Etude du phénomène de remontée de boue sur le décanteur lamellaire de la station de traitement d'eau de Paspanga au Burkina Faso
}

\author{
Elisabeth R. OUEDRAOGO ${ }^{1}$, Crépin I. DIBALA ${ }^{1 *}$, Kiessoun KONATE ${ }^{1}$, \\ Jean DIARRA ${ }^{2}$ et Mamoudou H. DICKO ${ }^{1}$
}
${ }^{1}$ Laboratoire de Biochimie, Biotechnologie, Technologie Alimentaire et Nutrition (LABIOTAN), Université Joseph KI-ZERBO de Ouagadougou, 03 BP: 848, Ouagadougou 03, Burkina Faso.
${ }^{2}$ Laboratoire Central de l'Office National d'Eau et d'Assainissement. *Corresponding author; E-mail: dibalacrepin@yahoo.fr; Phone: 0022670141596

\section{RESUME}

L'eau potable, un aliment de base et son accès est l'un des besoins humains fondamentaux. A Ouagadougou, l'eau potable provient du traitement des eaux de surface à Paspanga et à Ziga. Les remontées de boues constituent un phénomène persistant dans les processus de traitement de l'eau à Paspanga. L'objectif de ce travail était de trouver les causes de ce phénomène afin d'optimiser les coûts et taux de production d'eau potable. Pour ce faire, les paramètres physico-chimiques et des essais de traitements de l'eau brute ont été réalisés selon les normes de qualité. Les résultats ont montré que l'eau est potabilisable. En effet, la turbidité, la teneur en matière en suspension et en matière organique sont respectivement en moyenne 116,52 NTU, 10,85 mg/l, 4,72 $\mathrm{mg} / \mathrm{l} \mathrm{d} \mathrm{O}_{2}$ en mai et 719,13 NTU, $21,07 \mathrm{mg} / \mathrm{l}, 13,08 \mathrm{mg} / \mathrm{l} \mathrm{d} \mathrm{O}_{2}$ de juin à juillet. Le $\mathrm{pH}$ de l'eau en mai est faible et en moyenne 6,89 et 7,50 dans les mois de juin à juillet. La mise en évidence de la production de gaz révèle que cette production est progressive. Les remontées de boues sont donc une résultante de deux facteurs : la qualité de l'eau brute et la production de gaz au fond des décanteurs.

(C) 2020 International Formulae Group. All rights reserved.

Mots clés : Décanteur, remontée de boue, traitement, eau

\section{Study of the phenomenon of mud increase on the lamellate decanter of the station of treatment of water of Paspanga in Burkina Faso}

\begin{abstract}
Drinking water is a basic food and its access is one of the fundamental human needs. For Ouagadougou, it comes from the station of water treatment of surface at Paspanga and Ziga. The mud increase constitutes a persistent phenomenon in the treatments of water of Paspanga. The objective of this work was to find the causes of this phenomenon in order to optimize the costs and rate of drinking water production. To be done, the physicochemical parameters and the tests of rough water treatments were carried out according to standards of quality. The results showed that water is potabilisable. Indeed, the average of turbidity, content of organic matter and suspended matter of water were respectively $116,52 \mathrm{NTU}, 10,85 \mathrm{mg} / \mathrm{l}, 4,72 \mathrm{mg} / \mathrm{l}$ of $\mathrm{O}_{2}$ in May and 719,13 NTU, 21,07 mg/l, 13,08 $\mathrm{O}_{2} \mathrm{mg} / \mathrm{l}$ from June to July. The $\mathrm{pH}$ of water varied to 6,89 in May; to 7.50 in June and July. In addition, the results showed that the gas production revealed is progressive over time, which would mean that the mud increased would be a result of two factors: the quality of raw water and the production of gas at the bottom of the decanters.
\end{abstract}

(C) 2020 International Formulae Group. All rights reserved.

Keywords: Decanter, rising mud, treatment, water 


\section{INTRODUCTION}

L'eau est une ressource indispensable à la vie et aux activités humaines. "Nous en avons besoin pour la santé, la sécurité alimentaire et le progrès économique », a déclaré Ban Ki-moon, Secrétaire général de l'ONU (Sommet de l'eau, 2013). Cependant à l'échelle mondiale, le volume globale d'eau douce est estimé à environ à $0,76 \%$, les océans $97,5 \%$ et $1,74 \%$ pour les glaciers polaires. Pour faire face à cette rareté naturelle de l'eau et répondre aux besoins croissants en eau douce, plusieurs retenues d'eau ont été construites au Burkina Faso.

Au Burkina Faso, l'eau potable est un enjeu important. Environ 780 millions de personnes, soit $11 \%$ de la population mondiale n'ont pas accès à l'eau potable et la plupart d'entre elles vivent en Afrique sub-saharienne (OMS, 2012). En effet 4 milliards de maladies diarrhéiques se produisent chaque année, dont $88 \%$ sont directement associées à une eau insalubre (OMS, 2007). Le Burkina Faso, dans l'atteinte des Objectifs Millénaire du Développement pour le secteur de l'Approvisionnement en Eau Potable et d'Assainissement, a élaboré le Programme National d'Approvisionnement en Eau Potable et d'Assainissement. La production et la distribution de l'eau dans les centres urbains au Burkina Faso ont été déléguées par l'Etat à l'Office National de l'Eau et de l'Assainissement (ONEA). Pour la ville de Ouagadougou, elle exploite principalement des eaux de surface provenant de deux grandes retenues d'eau (les barrages Ouaga 3 et Loumbila). Cependant, ces eaux de surface contiennent généralement une grande quantité de solides en suspension, bactéries, algues, matière organique (Dicko, 2016 ; Njoyim et al., 2016). Pourtant la consommation d'une eau de mauvaise qualité est la principale cause de nombreuses maladies qui entraînent de nombreux décès surtout chez les enfants en bas âge (OMS, 2017). Il est donc est nécessaire d'effectuer un traitement de cette eau insalubre avant toute consommation et assurer une production suffisante à toute la population.

Le processus de traitement d'eau comprend une phase de clarification qui génère des flocs sous forme de boues. Ces flocs formés pour être précipités au fond des décanteurs, pour des raisons fortes variées, se retrouvent à un moment donné, soumis à des mouvements ascensionnels appelés remontées de boues (Tidiani, 2017) qui occasionnent de graves perturbations de la chaîne de traitement. En 1985, le comité Interafricain d'étude Hydraulique a effectué sur les stations de traitement d'eau à Ouagadougou et Koudougou, une étude descriptive du phénomène qui constituait déjà un premier outil d'orientation à la décision pour l'élaboration d'un projet de recherche. Trentedeux ans plus tard, ce problème n'a toujours pas eu de solution. En effet, l'exploitation des rapports annuels et des registres de suivi de traitement d'eau à la station de Paspanga a mis en évidence une dégradation continue des rendements de la station et une fréquence élevée de lavage des filtres depuis 2012 contribuant ainsi à l'augmentation du coût de traitement de l'eau et la baisse du taux de traitement. Ce constat est plus accentué dans la période de mars à juillet au cours desquelles les remontées de boues ont été le plus importantes. L'une des solutions pour pallier aux remontées de boues était de couvrir les décanteurs et les filtres de la nouvelle station de Ziga et l'Unité Compact de Degremont à Paspanga. Malgré ces investissements, ce phénomène persiste. C'est dans ce contexte que s'inscrit ce travail dont l'objectif était de rechercher les causes des remontées de boues qui perturbent la chaine de traitement d'eau afin d'optimiser les taux et coûts de production d'eau potable dans les stations de traitement d'eau au Burkina Faso.

\section{MATERIEL ET METHODES}

Matériel d'étude

\section{Station de traitement d'eau de Paspanga}

La station de traitement des eaux de surface de Paspanga est située au centre de la ville de Ouagadougou et alimente environ 30 communes de la ville en eau potable. Elle traite les eaux brutes provenant des barrages de Loumbila et du barrage $n^{\circ} 3$ de Ouagadougou. Elle a une capacité de $50600 \mathrm{~m}^{3} / \mathrm{j}$ afin de satisfaire $30 \%$ des besoins de la ville. La station 
de traitement de ZIGA située à $60 \mathrm{~km}$ de la ville s'occupe des $70 \%$ de la desserte.

\section{Prélèvement et conservation des échantillons d'eau brute \\ L'eau brute utilisée constituée} d'éléments minéraux et organiques, est prélevée à l'entrée de la station pendant la période allant de mai à juillet 2017 selon un plan d'échantillonnage. Les prélèvements d'eau de barrage à l'entrée de la station se justifient par la nécessité d'aligner l'étude expérimentale aux réalités de la station. En effet, les prélèvements faits sur le plan d'eau pourraient biaiser les résultats des analyses de turbidités, de matière organique et de certains paramètres physiques. De plus, les prélèvements à l'entrée de la station pourraient également permettre de mieux observer les phénomènes physiques liés à l'eau et aux débits d'exploitation.

Un total de 36 échantillons d'eau provenant des barrages $\mathrm{N}^{\circ} 3$ de la ville de Ouagadougou (Ouaga 3), de Loumbila ont été utilisés. Trois échantillons d'eau de $5 \mathrm{~L}$ ont été prélevés, étiquetés chaque fois aux environs de $8 \mathrm{~h}$ durant douze (12) semaines selon les normes ISO 5667-1 et ISO 5667-2 (2007), puis conservés et transportés au laboratoire conformément à la norme française NF EN ISO 5667-3 (2004). Chaque flacon de $5 \mathrm{~L}$ nettoyé est rincé trois fois avec l'échantillon à prélever de façon à conserver une certaine représentativité de l'échantillon. Le reste des échantillons d'eau est conservé au réfrigérateur.

\section{Échantillons de boues de traitement}

Les boues de traitement issues de nos jar-test ont été utilisées pour sa caractérisation physico-chimique. La boue de traitement est collectionnée après le jar -test dans une bouteille ambrée stérile puis utilisée pour la mesure de l'indice de boue; l'estimation de la production de gaz par les boues au fond du décanteur et la $\mathrm{DBO}_{5}$.
Méthodes

Détermination des paramètres physicochimiques d'eau brute et de la boue de traitement

Mesure du $\mathrm{pH}$, turbidité, conductivité, température : Le $\mathrm{pH}$ a été mesuré suivant une méthode électrochimique à l'aide d'un $\mathrm{pH}$ mètre/ thermomètre WWTW INOLAB pH-730 équipé d'une électrode combinée Sen Tix 41 conformément à la NFT 90-008. La turbidité a été mesurée par méthode néphélométrique avec un appareil turbidimètre WTW turb 550 selon la NF T90-033 et la conductivité, par la méthode électrochimique selon la NFT90-03 à $25^{\circ} \mathrm{C}$ à l'aide d'un conductimètre CDM 83. La température a été déterminée par méthode électrochimique, à l'aide d'un conductimètre CDM83 conformément à la NFT90-031 (Tapsoba, 2016).

\section{Mesure des matières en suspension (MES)}

Les matières en suspension ont été mesurées par pesée après filtration sur une membrane de 0,45 $\mu \mathrm{m}$ de porosité de masse $\left(\mathrm{M}_{0}\right)$ d'un volume d'eau connu $(\mathrm{V})$ sur une pompe Sartorius 16612. On récupère le filtre mouillé et on le sèche dans l'étuve pendant 2 heures à $105^{\circ} \mathrm{C} \pm 2{ }^{\circ} \mathrm{C}$ dans une étuve Binder GMBH 14D-78532 Tuhlingen, selon la méthode NF T90-105-2 puis on pèse le filtre avec MES $\left(\mathrm{M}_{1}\right)$, la formule de calcul suivante permet la détermination des MES :

$$
\text { MES = (M1 -M0) } / \mathrm{V} * 1000 .
$$

La teneur des matières en suspension est exprimée en $\mathrm{mg} / \mathrm{l}$.

Détermination de matière organique dans l'eau brute: l'oxydabilité au permanganate de potassium $\left(\mathrm{KMnO}_{4}\right)$

Le taux de $\mathrm{KMnO}_{4}$ a été déterminé par oxydation chimique à chaud en milieu acide. L'eau est portée à ébullition en présence d'une solution de permanganate de potassium à N/80 dont l'excès est dosé après 10 minutes d'ébullition. La différence du volume à analyser et du volume de $\mathrm{KMnO} 4$ utilisé pour le titrage de l'eau distillée (témoin) a donné la teneur de $\mathrm{KMnO}_{4}$ en mg $\mathrm{O}_{2} / \mathrm{l}$. Oxydabilité au $\mathrm{KMnO}_{4}\left(\mathrm{mgO}_{2} / \mathrm{l}\right)=($ titrage-blanc $)$. 


\section{Mesure de la $\mathrm{DBO}_{5}$ (Demande Biochimique en Oxygène)}

La Mesure de la $\mathrm{DBO}_{5}$ de l'eau brute ou de boue a été réalisée par un système Oxitop qui mesure l'oxygène consommé par les microorganismes et mémorise une valeur toutes les $24 \mathrm{~h}$ pendant 5 jours (Sawadogo, 2012).

\section{Dosage des nitrites, nitrates et orthophosphates}

Les concentrations de nitrites ont été déterminées par spectrophotométrie d'absorption moléculaire. La lecture a été faite après vingt minutes d'incubation avec le nitriver5 à l'aide d'un spectrophotomètre DR 3800. L'absorbance a été lue à $371 \mathrm{~nm}$. La valeur est exprimée en $\mathrm{mg} / \mathrm{l}$ de $\mathrm{NO}_{2}$.

Le dosage des nitrates dans les eaux a également été réalisé par spectrophotométrie d'absorption. La lecture a été faite après cinq minutes d'incubation avec le nitraver5 à l'aide d'un spectrophotomètre DR 3800. L'absorbance a été lue à $355 \mathrm{~nm}$. La valeur est exprimée en $\mathrm{mg} / \mathrm{l}$ de $\mathrm{NO}_{3}$.

Le dosage des orthophosphates dans les eaux a été réalisé par spectrophotométrie d'absorption moléculaire. La lecture a été faite après deux minutes d'incubation avec le phosphover3 à l'aide d'un spectrophotomètre DR 3800. L'absorbance a été lue à $490 \mathrm{~nm}$. La valeur est exprimée en $\mathrm{mg} / \mathrm{l}$ de $\mathrm{PO}_{4}{ }^{3}$.

\section{Essai de traitement d'eau brute et mise en évidence du phénomène biologique sur les émissions de gaz \\ Le jar test est une méthode qui consiste} à déterminer à l'échelle laboratoire, les doses optimales de coagulant et de floculant qui seront injectées à l'usine. Remplir six béchers avec chacun 1 litre d'eau à traiter. Ajouter dans les béchers des doses variables de coagulant; après l'addition du coagulant, régler la vitesse de rotation des hélices $120 \mathrm{trs} / \mathrm{min}$ pour un mélange rapide d'une durée de $3 \mathrm{~min}$. C'est la phase de de coagulation. Ajouter le floculant dans chaque bécher à une dose constante et régler la vitesse de rotation des hélices à 40 trs/min pour la formation des flocs. Le mélange lent permet aux flocs de se rencontrer pour une agglomération: C'est la phase de floculation. Après la floculation, arrêter les hélices et laisser les flocs formés se décanter pendant 10 $\mathrm{mn}$. La turbidité du surnagent a été ensuite mesurée. Les doses optimales ont été obtenues avec le surnagent le plus limpide. Ces doses sont ensuite converties à l'échelle station et seront appliquées pour le traitement des eaux brutes qui arrivent dans l'unité.

L'application industrielle des résultats du Jar test se fera par la mise en œuvre des étapes suivantes :

Calcul de la quantité de produit chimique $(\mathrm{m})$ consommée en une unité de temps $(\mathrm{en} g / \mathrm{h}) \mathrm{m}=\left(\mathrm{C}_{\mathrm{s}} \times \mathrm{Q}\right)$ en gramme par heure

$\mathbf{C}_{\mathrm{s}}=$ quantité de produit chimique en grammes nécessaires pour un mètre cube (Obtenue par l'essai de traitement); $(\mathrm{g} / \mathrm{m} 3)$.

$\mathbf{Q}=$ débit d'eau à traiter par unité de temps ; (m3/ h) fixé par le responsable de la station.

Calcul du débit $\mathrm{q}$ de la pompe doseuse à fixer : $\mathrm{q}(\mathrm{L} / \mathrm{h})=\mathrm{m} / \mathrm{C}=\underline{\left(\mathrm{C}_{\mathrm{s}}\right.}$ $\underline{\mathrm{x} Q}) / \mathrm{C}_{\mathrm{g} / 1}$

\section{Réglage de la pompe doseuse et du débit d'eau dans le décanteur}

Le réglage de la pompe doseuse et le débit d'eau sera fait par le responsable de la station de traitement. Le processus sera mis en observation et les phénomènes associé aux remontées de boue seront enregistrés. Le suivi des remontées de boues sur le décanteur est effectué par contrôle et observation puis reportés sur des fiches de collection de données durant toute la période selon des critères définis.

\section{Mise en évidence du phénomène biologique sur les émissions de gaz}

La dégradation de la matière organique produit des gaz selon un processus aérobie ou anaérobie elle pourrait être similaire à ce qui se passe au fond des décanteurs. Les flacons ont été assimilés à des fermenteurs et la production de gaz a été suivie d'une déformation du flacon qui se gonfle. L'émission des gaz par les boues de traitement est suivie et reportée sur une fiche durant toute la période d'étude.

\section{Caractérisation des boues de traitement}

Les boues issues des jar-tests sont recueillies dans des flacons plastiques pour 
déterminer deux paramètres (mesure de la $\mathrm{DBO}_{5}$ et de l'indice de Mohlman)

\section{Mesure de l'indice de Mohlman (IM)}

Introduire dans une éprouvette 1 litre de boues de traitement issu du jar-test, puis, déterminer le volume (en $\mathrm{ml}$ ) occupé par les boues après décantation d'une demi-heure. Rechercher ensuite la teneur en matières sèches d'un litre de boues à analyser. L'indice de Mohlman est représenté par le rapport :

$\mathrm{IM}=$ Indice de décantation $(\mathrm{m} h) /($ Masse de la matière sèche $(\mathrm{g} 1)$

\section{Suivi de remontées de boues}

L'exhaure d'eau brute est suivie très souvent de prise d'air qui s'introduisent dans les conduites. Ainsi, dans les projets d'AEP, on prévoit des ventouses qui ont pour rôle essentiel de vidange ou de purger l'air contenu dans les conduites au cours du transport d'eau. La mise en évidence de l'incidence de l'état des organes du réseau sur les processus de décantation est effectuée par contrôle et observation puis reportée sur des fiches de collection de données durant toute la période selon des critères définis en fonction du paramètre suivi. Les données recueillies sont ensuite analysées afin de tirer une conclusion sur la cause réelle du phénomène.

\section{Traitement des données}

Les données ont été saisies et traitées avec un logiciel Microsoft Word 2016 pour la narration et avec un logiciel Excel 2016 pour les tableaux et les graphiques. Le logiciel XLSTAT (Version 2016) a été utilisé pour l'analyse des variances (ANOVA) afin de comparer les valeurs moyennes des différentes variables. Le test Turkey a été utilisé pour déterminer les différences significatives entre les variables au seuil de $5 \%$.

\section{RESULTATS}

\section{Paramètres physico-chimiques de l'eau brute}

L'évolution du $\mathrm{pH}$ au cours de la période d'étude varie du $\mathrm{pH}$ minimum de 6,55 et un maximum de 7,88 , soit une moyenne de 7,2 . On obtient une forte variabilité du $\mathrm{pH}$ au cours de la période d'étude $(\mathrm{Var}=0,3)$. Ce $\mathrm{pH}$ a connu une très faible évolution à tendance alcaline dans les cinq premières semaines (variance 0,02) qui correspondent à la période sèche. Le $\mathrm{pH}$ de l'eau est resté dans la plage recommandée pour une bonne coagulation entre 6,5 à 7,5.

Les résultats de l'évolution de la turbidité indiquent que la période d'étude a été marquée par des valeurs faibles de turbidité de la semaine $S_{1}$ à la semaine $S_{5}$. La plus forte turbidité a été enregistrée à la huitième semaine $\mathrm{S}_{8}$ (1076,8 NTU). La plus faible valeur de la turbidité (19,34 NTU) est observée au cours de la semaine $S_{1}$ du mois de mai

Les résultats de l'évolution de la conductivité montrent une faible minéralisation de l'eau brute traitée au cours des cinq premières semaines et accroissement de la conductivité de l'eau à partir de la semaine $\mathrm{S}_{6}$.

Le suivi de l'évolution de la température de l'eau brute révèle une chute de la température passant de $33,4{ }^{\circ} \mathrm{C}$ à $28,5^{\circ} \mathrm{C}$ de la semaine $S_{1}$ à la semaine $S_{3}$ au cours du mois de mai. Les valeurs n'ont pas connu une grande évolution et sont faibles au cours des mois de juin et juillet.

Les valeurs des nitrites ont considérablement baissé de la semaine $S_{1}$ à la semaine $S_{7}$. Par contre, on note un accroissement des teneurs de la semaine $S_{1}$ à la semaine $S_{11}$. La plus forte valeur $(0,157 \mathrm{mg} / \mathrm{l})$ a été observé au cours de la semaine $S_{1}$ du mois de mai et la plus faible valeur $(0,063 \mathrm{mg} / \mathrm{l})$ au cours de la semaine $S_{9}$ du mois de juillet.

Pour les nitrates, les valeurs varient de la semaine $S_{7}$ à la semaine $S_{11}$. La plus forte valeur $(27,415 \mathrm{mg} / \mathrm{l})$ a été observée au cours de la semaine $S_{9}$ et la plus faible valeur $(11,403$ $\mathrm{mg} / \mathrm{l}$ ) au cours de la semaine $\mathrm{S}_{1}$. Les valeurs des nitrates ont considérablement augmenté de la semaine $S_{6}$ à la semaine $S_{9}$. Ces paramètres physico-chimiques sont représentés par le Tableau 1.

\section{Essai de traitement d'eau brute}

Le Tableau 2 illustre les résultats de Jartest pour les doses applicables en traitement. Le taux minimal de sulfate d'alumine au cours de 
la période d'étude est de $20 \mathrm{mg} / \mathrm{l}$ et le maximum à $65 \mathrm{mg} / \mathrm{l}$ à la semaine $\mathrm{S}_{10}$. L'eau clarifiée au cours de la période d'étude a une turbidité d'environ $3,89 \mathrm{NTU}, 1,59 \mathrm{mg} / 1$ d' $\mathrm{O}_{2}$ de matière organique et un $\mathrm{pH}$ de 6,84 . L'application de ces valeurs à l'échelle industrielle donne respectivement pour le mois de mai, juin et juillet, 135 litres par heure, 292 litres par heure et 247 litres par heure avec un débit d'exhaure de $675 \mathrm{~m}^{3} / \mathrm{h}$ en mai et de 450 $\mathrm{m}^{3} / \mathrm{h}$ au cours du mois de juin et juillet inférieur au débit nominal du décanteur. La concentration de sulfate dans le bac est restée constante à $100 \mathrm{~g} /$ litre au cours de la période d'étude.

\section{Mise en évidence du phénomène biologique sur les émissions de gaz}

Le suivi de la boue placée dans l'enceinte hermétiquement fermée a permis d'observer des gouttelettes d'eau sur les parois du flacon et de flocs qui remontent à l'intérieur du récipient. Au bout de 35 jours, l'enceinte se gonfle et nous notons des sifflements à l'ouverture de l'enceinte après cinq semaines qui indiquent l'émission des gaz produit. Le suivi de cette émission est consigné dans le Tableau 3.

\section{Caractérisation des boues de traitement Demande Biochimique en Oxygène $\left(\mathrm{DBO}_{5}\right)$}

Les résultats de la Demande Biochimique en Oxygène indique une faible évolution de ce paramètre de la semaine $S_{1}$ à la semaine $S_{7}$ puis une évolution importante de la semaine $S_{8}$ à la semaine $S_{12}$. Les boues contiennent donc de la matière organique biodégradable (Figure 1).

\section{Indice de Molhman ou Indice de boue}

Les résultats des tests de décantation en éprouvette donnent des valeurs qui oscillent entre 49,5 et $132,3 \mathrm{ml} / \mathrm{g}$. Les boues de la semaine $S_{1}$ à la semaine $S_{5}$ sont moins aptes à la sédimentation comparativement aux boues de la semaine $\mathrm{S}_{6}$ à la semaine $\mathrm{S}_{12}$ (Figure 2).

\section{Suivi des remontées de boues}

Les intensités des remontées de boues au cours de notre période d'étude sont consignées dans la Figure 3 . L'intensité de boue est très forte de la semaine $S_{1}$ à la semaine $\mathrm{S}_{8}$. Elle est moyenne entre la semaine $\mathrm{S}_{9}$ et la semaine $S_{10}$ et faible entre $S_{11}$ et $S_{12}$ (Figure 3).

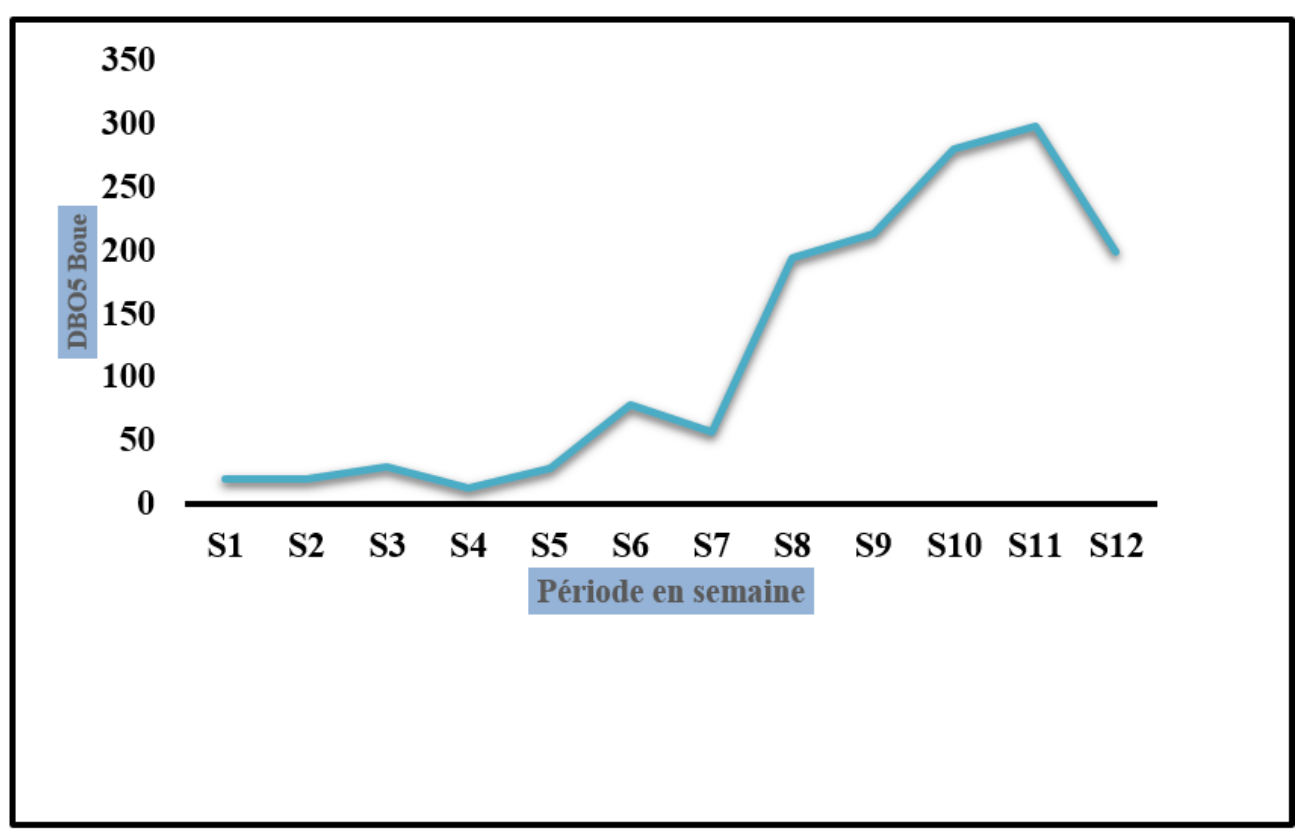

Figure 1 : Evolution de $\mathrm{la}_{\mathrm{DBO}}$ des boues de traitement de mai à juillet 2017. 
Tableau 1 : Paramètres physicochimiques de l'eau brute de la station durant la période d'étude.

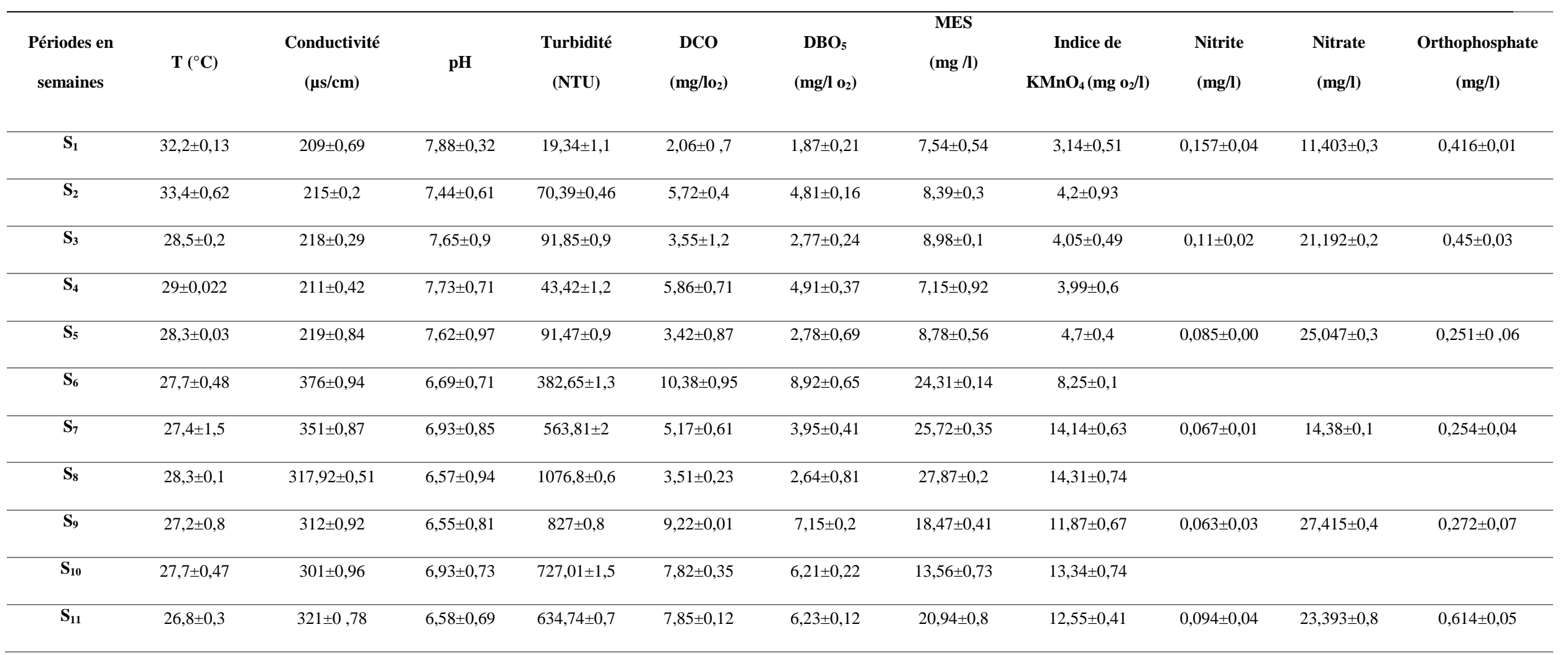


E. R. OUEDRAOGO et al. / Int. J. Biol. Chem. Sci. 14(2): 364-377, 2020

\begin{tabular}{|c|c|c|c|c|c|c|c|c|c|c|c|}
\hline$S_{12}$ & $27,4 \pm 0,83$ & $290 \pm 0,81$ & $7,82 \pm 0,21$ & $485,47 \pm 0,6$ & $7,25 \pm 0,52$ & $6,83 \pm 0,36$ & $19,86 \pm 0,73$ & $12,32 \pm 0,64$ & & & \\
\hline Moyenne & $28,66 \pm 0,44$ & $226,57 \pm 0.66$ & $7,20 \pm 0,70$ & $417,83 \pm 0,9$ & $5,98 \pm 0,53$ & $4.92 \pm 0,37$ & $15,96 \pm 0,40$ & $5,74 \pm 0,57$ & $0,10 \pm 0,00$ & $20,4 \pm 0,17$ & $0,38 \pm 0,02$ \\
\hline Maxima & $33,4 \pm 0,62$ & $376 \pm 0,94$ & $7,88 \pm 0,3$ & $1076,8 \pm 0,6$ & $10,38 \pm 0,95$ & $8,92 \pm 0,65$ & $27,87 \pm 0,2$ & $14,31 \pm 0,74$ & $0,157 \pm 0,04$ & $27,415 \pm 0$ & $0,614 \pm 0,5$ \\
\hline Minima & $26,8 \pm 0,3$ & $209 \pm 0,69$ & $6,55 \pm 0,8$ & $19,34 \pm$ & $2,06 \pm 0,7$ & $1.87 \pm 0,21$ & $7,15 \pm 0,92$ & $3,14 \pm 0,51$ & $0,063 \pm 0,03$ & $11,403 \pm 40,3$ & $0,251 \pm 0,6$ \\
\hline Variance & 4,2 & 48,3 & 0,3 & 62,6 & 6,6 & 2,95 & 60,5 & 3,1 & 0,0 & 39,5 & 0,0 \\
\hline $\begin{array}{c}\text { Norme BF } \\
2011\end{array}$ & $18-40$ & 1000 & $6,5-8,5$ & - & - & - & 25 & - & 0,2 & 50 & 0,7 \\
\hline
\end{tabular}

S : semaine 
Tableau 2 : Essai de traitement.

\begin{tabular}{|c|c|c|c|c|c|c|c|c|c|c|c|}
\hline SEMAINES & 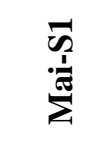 & 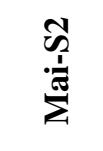 & 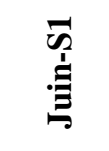 & 令 & 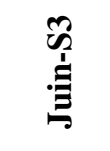 & 䓛 & 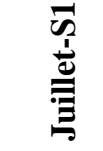 & 先 & 里 & 落 & 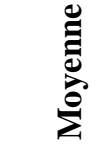 \\
\hline $\begin{array}{c}\text { Sulfate d'alumine } \\
(\mathrm{mg} / \mathrm{l})\end{array}$ & 25 & 20 & 20 & 30 & 45 & 55 & 55 & 65 & 55 & 50 & 35,5 \\
\hline Synthofloc (mg/l) & 0,1 & 0,1 & 0,1 & 0,1 & 0,1 & 0,1 & 0,1 & 0,1 & 0,1 & 0,1 & 0,1 \\
\hline Turbidité (NTU) & 4,21 & 3,97 & 4,75 & 5,52 & 3,03 & 2,46 & 4,47 & 2,53 & 2,95 & 5,04 & 3,89 \\
\hline pH & 7,51 & 7,35 & 7,14 & 6,55 & 6,62 & 6,5 & 6,5 & 6,54 & 6,52 & 7,23 & 6,84 \\
\hline MO (mg/l d'O2) & 1,2 & 1,1 & 1,4 & 2,8 & 2,2 & 1,9 & 1,6 & 1,2 & 1,1 & 1,4 & 1,59 \\
\hline $\begin{array}{c}\text { Rendement } \\
\text { d'élimination de } \\
\text { la MO }(\%)\end{array}$ & 61,7 & 94,3 & 94,8 & 98,6 & 99,4 & 99,7 & 99,4 & 99,4 & 99,5 & 98,9 & 84,62 \\
\hline $\begin{array}{l}\text { Rendement } \\
\text { d'élimination } \\
\text { turbidité }(\%)\end{array}$ & 78,2 & 73,8 & 70,2 & 66,1 & 69,2 & 77,1 & 72,7 & 83,6 & 80,2 & 77,8 & 74,48 \\
\hline
\end{tabular}

Tableau 3 : Suivi de l'émission des gaz par les boues de traitement.

\begin{tabular}{lllllll}
\hline Période & FS $_{1}$ & FS $_{3}$ & FS $_{5}$ & FS $_{7}$ & FS $_{9}$ & FS $_{11}$ \\
\hline $\begin{array}{l}\text { Date de mise en } \\
\text { flacon }\end{array}$ & $\mathbf{8 / 0 5 / 2 0 1 8}$ & $\mathbf{2 2 / 0 5 / 2 0 1 8}$ & $\mathbf{5 / 0 6 / 2 0 1 8}$ & $\mathbf{1 9 / / 0 6 / 2 0 1 8}$ & $\mathbf{3 / 0 7 / 2 0 1 8}$ & $\mathbf{1 7 / 0 7 / 2 0 1 8}$
\end{tabular}

\section{Phénomène}

observé au

cours de la

conservation

de

l'échantillon

\begin{tabular}{|c|c|c|c|c|c|c|}
\hline $\begin{array}{l}\text { Flacon ouvert } \\
\text { et observation }\end{array}$ & - & - & - & $\mathbf{F S}_{1}$ & $\mathbf{F S}_{3}$ & $\mathbf{F S}_{4}$ \\
\hline $\begin{array}{l}\text { Intensité du } \\
\text { phénomène } \\
\text { observé }\end{array}$ & - & - & - & 3 & 2 & 3 \\
\hline
\end{tabular}

Bulles d'air sur les parois supérieures du flacon au bout d'une semaine suivi d'une remontée de boue dans le flacon puis sa déformation.
Très faible : 1 ; Faible : 2 ; Moyenne : 3 ; Accentuée : 4 ; Très accentuée : 5 FS = flacon de la semaine. 


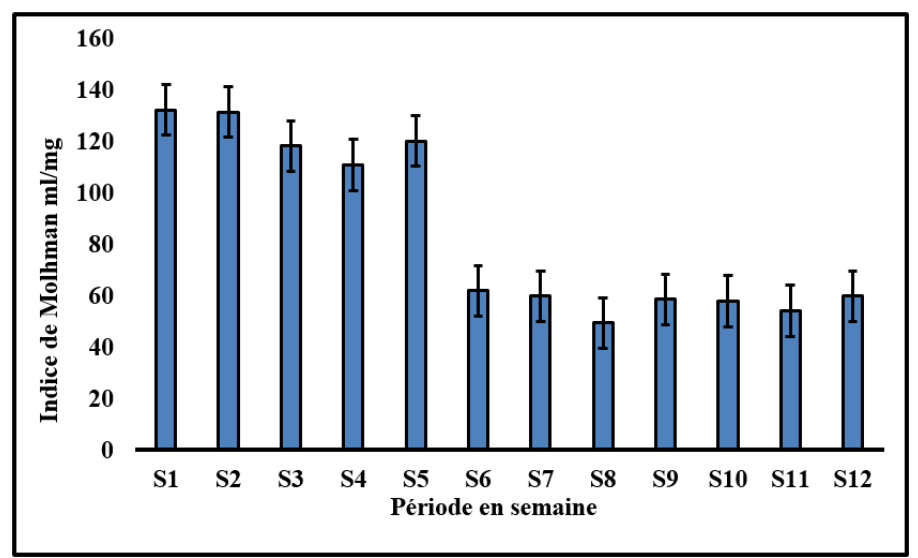

Figure 2: Evolution de l'aptitude des boues de traitement à la décantation de mai à juillet 2017.

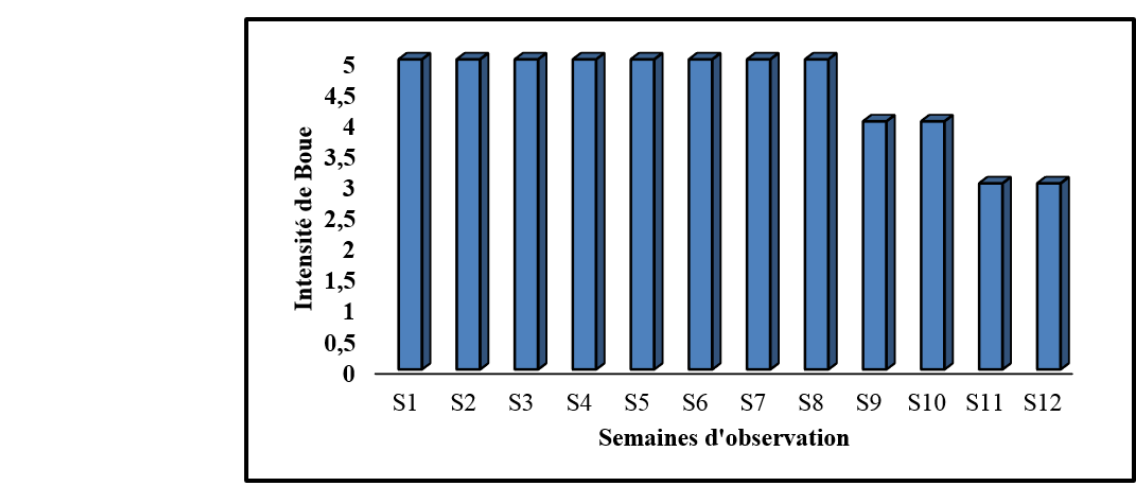

0 : pas de boue $; 1$ : très peu de boue $; 2$ : peu de boue $; 3$ : moyenne $4 ;$ abondante $; 5:$ très abondante : Grille d'évaluation.

Figure 3 : Evolution de l'intensité des remontées de boues de mai à juillet 2017.

\section{DISCUSSION}

Les paramètres physico-chimiques de l'eau brute utilisée pour la potabilisation au cours des mois de mai, juin et juillet 2017 montrent que : les pH ont évolué vers l'acidité avec l'arrivée des pluies. En effet, dès la sixième semaine (en mi-juin), le $\mathrm{pH}$ est passé de 7,62 à 6,69; cela est dû surtout à l'apport en polluant organique dans le barrage. Cet apport influe significativement sur le traitement. En effet, la coagulation-floculation est fortement liée au $\mathrm{pH}$. Toutefois, la dégradation du $\mathrm{pH}$ avec l'arrivée des pluies est suivie de l'utilisation de la chaux pour la mise à l'équilibre des eaux. Le $\mathrm{pH}$ des eaux du barrage Ouaga 3 et de Loumbila permet la formation de flocs aptes à la décantation car les valeurs de $\mathrm{pH}$ ont fluctué dans la zone de $\mathrm{pH}$ optimal compris entre 5,8 - 7,2 pour la formation du précipité d'aluminium $\left(\mathrm{Al}_{2} \mathrm{OH}\right)_{3}$ responsables de la coalescence des colloïdes lors de la coagulation qui permet la formation de flocs aptes à la décantation lors de la floculation (Mujawamariya, 2012). Les valeurs de $\mathrm{pH}$ ont également fluctué autour des valeurs recommandées par l'OMS (2011) pour les eaux potabilisables et la norme nationale pour les eaux destinées à la potabilisation. Nos résultats corroborent à ceux obtenus par Tapsoba (2016) qui trouvaient que les valeurs de $\mathrm{pH}$ enregistrées de septembre à décembre s'accordent avec les valeurs recommandées par l'OMS (2011) pour les eaux usées et par Rodier (2009).

Les données de la turbidité indiquent que la période d'étude a été marquée par des valeurs faibles de turbidité de la semaine $S_{1}$ à la semaine $\mathrm{S}_{5}$ s'expliquant ainsi par la décantation naturelle de l'eau et le non apport en matière en suspension dans le barrage. La 
plus forte turbidité a été enregistrée à la huitième semaine $\left(\mathrm{S}_{8}\right)$ due à l'arrivée de la pluie qui a occasionné l'apport de matières exogènes dans les retenues d'eau. Selon Hernández De León (2006), les fortes turbidités favorisent une meilleure décantation. Des résultats similaires ont été obtenus par Ouattara (2016) sur les eaux de Loumbila et Ouaga 3.

La conductivité d'une eau traduit son degré de minéralisation. L'évolution de la conductivité montre une faible minéralisation de l'eau au cours des cinq premières semaines. Cette tendance s'explique par le fait que les particules minérales sont plus aptes à la décantation naturelle car elles sont de forte densité d'où une turbidité faible durant ces périodes. En effet Ballouki (2012), a justifié l'augmentation de la conductivité en période hivernale par l'apport de colloïdes minérales dans la retenue et cela permet une bonne coagulation lors du traitement de l'eau.

Les fortes températures de l'eau enregistrées au cours du mois de mai sont liées à la période marquée par de très hautes températures ambiantes qui impactent celles de l'eau dans les conduites. Pourtant une température basse en dessous de $7{ }^{\circ} \mathrm{C}$ ralentie la décantation du floc et la plage du $\mathrm{pH}$ optimal diminue selon Hernández De León (2006). Les températures de l'eau brute sont donc favorables à une meilleure décantation des flocs formés. L'ensoleillement accroîtrait l'activité photosynthétique dans le barrage et ainsi l'accroissement de la matière organique et la diminution de la turbidité colloïdale selon Lamizana-Diallo (2008). Il y aura donc une diminution de la concentration en particules colloïdales, moteurs de la coagulationfloculation car lorsque la concentration en particules colloïdales est faible, la probabilité de collision entre colloïdes est faible et il en résulte une mauvaise coagulation. $\mathrm{La}$ température de l'eau brute est conforme à la norme nationale des eaux destinées à la potabilisation (OMS, 2011).

La turbidité et la teneur en MES évoluent en synergie au cours de notre période d'étude. En effet, la turbidité et les MES de l'eau sont faibles de la semaine $\mathrm{S}_{1}$ à la semaine $S_{5}$. Ces faibles turbidités colloïdales et teneurs en MES rendent difficiles la coagulation floculation. Suite à la décantation naturelle de l'eau dans le barrage de la semaine $\mathrm{S}_{1}$ à la semaine $\mathrm{S}_{5}$, la turbidité s'augmente considérablement à partir de la semaine $\mathrm{S}_{6}$. Cette augmentation serait due à l'apport de grandes quantités de particules diverses en provenance des eaux de ruissellement orientées dans le barrage. Elles impactent significativement le traitement par l'utilisation de grande quantité de produits chimiques. En effet, la qualité de coagulation-floculation est fortement liée à la teneur en MES et la turbidité de l'eau brute. La valeur maximale recommandée pour le traitement d'eau de surface est de 1000 NTU pour la turbidité et 25 $\mathrm{mg} / \mathrm{l}$ pour les MES. Toutefois l'augmentation de ces teneurs avec l'arrivée des eaux de pluies est suivie de l'optimisation des quantités de produit pour une bonne élimination de ces matières. Au regard de l'évolution des MES et de la turbidité de l'eau, les analyses physiques révèlent que les eaux des barrages Ouaga 3 et Loumbila sont potabilisables durant la période car les valeurs de MES ont fluctué autour de valeurs recommandées par l'OMS (2011) et la norme nationale des eaux destinées à la potabilisation; mais les faibles turbidités et teneurs en MES impactent négativement la coagulation qui permet l'élimination des particules en suspension dans l'eau.

La teneur en nitrite de l'eau baisse de la semaine $S_{1}$ à la semaine $S_{7}$ tandis que la teneur en nitrate s'accroit dans l'eau au cours des trois premières semaines de la période des pluies, il y a donc eu un apport de matière azotée durant cette période. Elle s'explique par l'apport des nitrates des engrais azotés utilisés par les maraichers qui exploitent les terres avoisinantes et ensuite apportés par les eaux de ruissellement dans le barrage. Les valeurs enregistrées de nitrates et nitrites durant la période d'étude s'accordent avec les valeurs recommandées par l'OMS (2011) et la norme nationale pour les eaux à la potabilisation; mais ces valeurs sont supérieures à celles obtenues par Ouattara et al., (2012) dans les eaux du barrage de Ziga.

Les essais de traitements ont permis de faire le choix des valeurs optimales pour la clarification de l'eau à grande échelle. La formation et la stabilité des flocs dépendent du $\mathrm{pH}$ qui doit être maintenu dans les limites requises $(6,5$ à 8,5$)$. Le taux minimal de sulfate d'alumine au cours de la période d'étude est de $20 \mathrm{mg} / \mathrm{l}$ et le maximum à $65 \mathrm{mg} / \mathrm{l}$ à la semaine $\mathrm{S}_{10}$. L'eau clarifiée au cours de la période d'étude a une turbidité d'environ 3,89 NTU, $1,59 \mathrm{mg} / \mathrm{l} \mathrm{d} \mathrm{O}_{2}$ de matière organique et un $\mathrm{pH}$ 
de 6,84. L'application de ces valeurs à l'échelle industrielle donne respectivement pour le mois de mai, juin et juillet, 135 litres par heure, 292 litres par heure et 247 litres par heure avec un débit d'exhaure de $675 \mathrm{~m}^{3} / \mathrm{h}$ en mai et de 450 $\mathrm{m}^{3} / \mathrm{h}$ au cours du mois de juin et juillet inférieur au débit nominal du décanteur. La concentration de sulfate dans le bac est restée constante à $100 \mathrm{~g} /$ litre au cours de la période d'étude. Les jar-tests sont une simulation à petite échelle de ce qui se passe en station de traitement lors de la coagulation/floculation (Mujawamariya, 2012). Ces tests permettent d'évaluer différents paramètres tels que les doses de coagulant utilisés, le $\mathrm{pH}$ de l'eau et la turbidité optimale pour l'application industrielle lors du traitement de l'eau à grande échelle. En effet, une dose de coagulant excessive entraîne une forte acidification, une mauvaise turbidité de l'eau décantée puis augmentation du coût d'exploitation, tandis qu'une dose insuffisante conduit à une mauvaise qualité de l'eau traitée. La dose optimale de coagulant varie selon la qualité de l'eau brute. L'eau traitée en saison des pluies très chargée donne des flocons denses contrairement à l'eau brute de la saison sèche (Hernández De León, 2006).

Le rendement moyen de réduction de la turbidité varie entre $66,1 \%$ pour la semaine $\mathrm{S}_{2}$ du mois de juin et $83,6 \%$ pour la semaine $S_{2} d u$ mois de juillet. Quant à la matière organique, le rendement moyen de réduction est compris entre $61,7 \%$ pour la semaine $S_{1}$ de mai et $99,7 \%$ pour la semaine $S_{4}$ de juin. Le plus bas rendement de réduction de turbidité est enregistré dans le mois de juin ; cela est lié à une collision faible entre les particules colloïdales lors de la coagulation et il en résulte leur faible élimination. En effet, l'eau du barrage subit une décantation naturelle qui entraîne certaines particules colloïdales minérales chargées de l'eau dont le PM est élevé au fond du barrage. Par conséquent, l'eau arrive à la station à travers les conduites moins chargées en colloïdes et riches en particules organiques de faible PM et non chargées. Pourtant, la réaction qui conduit à la coalescence des colloïdes lors de la coagulation s'effectue entre les hydroxydes d'aluminium $\mathrm{Al}_{2}(\mathrm{OH})_{3}$ et les colloïdes chargés négativement (Mujawamariya, 2012); mais minoritaires dans l'eau d'où une mauvaise coagulation et des flocs moins denses avec des difficultés de décantation. Pour améliorer la qualité de l'eau pauvre en colloïdes à l'ONEA Paspanga. Ouattara (2016) avait utilisé la bentonite qui a permis d'obtenir les rendements de $98,20 \%$ de réduction de la turbidité en moyenne avec 40 $\mathrm{g} / \mathrm{l}$ de sulfate d'aluminium pour le mois de mai et $98,34 \%$ de réduction de la matière organique en moyenne pour le mois juin. En effet, le rendement de réduction de la matière organique peut atteindre $80 \%$ lorsque la bentonite est introduite après le coagulant sur les eaux Ouattara (2016). L'application des taux de traitement à l'échelle industrielle donne pour le mois de mai 135 litres par heure avec un débit d'exhaure de $675 \mathrm{~m}^{3} / \mathrm{h}$ tandis qu'en juin et juillet, le débit d'exhaure était de 450 $\mathrm{m}^{3} / \mathrm{h}$, mais la quantité d'eau produite est plus élevée en juin et juillet qu'en mai (292 l/h en juin et $247 \mathrm{l} / \mathrm{h}$ en juillet). Ces résultats s'expliquent par les pertes d'eau traitée due aux lavages des filtres très fréquents causés par les boues qui passent dans les filtres et entraînent rapidement leur colmatage.

Les résultats de la demande biochimique en oxygène confirment que les boues de traitement renferment des microorganismes aérobies. Ces boues renfermant une grande quantité de matière organique très biodégradable issues de la clarification de l'eau brute ; ces dernières subissent une dégradation lorsqu'elles séjournent dans les décanteurs de la surface jusqu'en profondeur.

Rodier (2005) et Souha et al (2017) ont montré que lorsque l'indice de Mohlman d'une boue est compris entre 50 et $150 \mathrm{ml} / \mathrm{g}$, elle est dotée d'une aptitude à la décantation. Le test d'aptitude de la boue à la décantation indique que l'indice de boue de la semaine $\mathrm{S}_{6}$ à la semaine $\mathrm{S}_{12}$ sont comprises entre $61,93 \mathrm{ml} / \mathrm{g}$ et $59,52 \mathrm{ml} / \mathrm{g}$; ces boues sont donc toutes aptes à la décantation. Cette période correspond à la saison pluvieuse et l'apport de MES dans le barrage augmente la charge colloïdale de l'eau et permet une bonne coagulation-floculation donc la formation de flocs plus denses. Cependant, les boues de la semaine $S_{1}$ à la semaine $S_{5}$ peuvent décanter mais sont moins aptes que celles de la période pluviale s'expliquant par la teneur faible de l'eau en colloïdes durant cette période.

L'eau de surface contient des gaz dissous de l'atmosphère, du sol et du sous-sol, de la dégradation des composés organiques azotés. Ces gaz, lors de l'exhaure, sont évacués par les ventouses placés sur les conduites d'exhaure (Nadjib, 2017). Malheureusement, 
ces organes ne fonctionnent plus. Ainsi au cours de l'exhaure se produit le phénomène de turbulence et l'eau en évoluant au sein de la conduite arrive avec des gaz emprisonnés qui ne trouvent un lieu pour s'échapper que sur le répartiteur et les décanteurs. Selon Moreau (2011), le reste des microbulles de gaz s'emprisonnent dans les flocons. Cela entraine des difficultés de décantation des flocs (Gaye, 2014).

Les boues formées ont une relation avec la qualité de l'eau traitée. Les remontées intenses durant cette période s'expliquent donc par la formation des flocs moins denses, moins aptes à la décantation qui sont plus soumis à l'action des forces de frottement qui s'opposent à leur décantation et les repoussent à la surface. A partir de la semaine $S_{9}$, les remontées de boues diminuent car cette période correspond à la tombée des pluies qui occasionnent les fortes turbidité et teneurs en MES favorisant ainsi une bonne coagulation et la formation de flocs plus denses qui résistent à la poussée des forces de frottement et aux gaz qui s'échappent sur le décanteur.

\section{Conclusion}

Notre étude avait pour objectif d'optimiser les taux et coûts de production d'eau potable à travers la recherche des causes de ce phénomène de remontée de boue afin de participer à l'atteinte de la sécurité alimentaire de la population du Burkina Faso. La caractérisation de l'eau brute a montré que les eaux du barrage de Loumbila et Ouaga 3 sont potabilisables mais leur traitement est très complexe en saison sèche à cause de leur pauvreté en colloïdes et leur richesse en particules organiques. L'analyse du procédé de traitement a révélé que la mauvaise décantation des flocs ne serait pas liée à la coagulationfloculation ni à l'application industrielle des essais. La caractérisation des boues issues du jar-test montre qu'elles sont en moyenne décantables mais plus aptes en saison pluvieuse et contiendraient des microorganismes qui dégradent la matière organique de ces boues. Les études expérimentales confirment que la dégradation des boues de traitement s'accompagne d'une production de gaz qui pourrait contribuer à pousser les flocs plus légers à la surface des décanteurs. Donc, on peut affirmer que les remontées de boues seraient la résultante de deux facteurs: la qualité de l'eau brute traitée et la conservation très prolongée des boues dans le fond des décanteurs entraînant des processus de fermentation.

\section{CONFLIT D'INTERETS}

Les auteurs déclarent qu'ils n'ont pas de conflit d'intérêts par rapport à cet article.

\section{CONTRIBUTIONS DES AUTEURS}

Tous les co-auteurs ont assuré la conduite de l'expérience et la rédaction du manuscrit.

\section{REMERCIEMENTS}

Nous remercions le laboratoire de l'ONEA pour l'assistance technique de ce travail et le projet RABIOTECH/ISP/ IPICS/SUEDE pour les frais de publication.

\section{REFERENCES}

Ballouki K. 2012. Etude de la qualité physicochimique et biologique de trois sources dans la régions de Midelt (Haut Moulouya). Mémoire de master de l'Université Sidi Mohammed Ben Abdellah, $76 \mathrm{p}$.

Dicko D. 2016. Etude de l'influence des caractéristiques de l'eau brute et de l'eau traitée sur l'efficacité de la chloration : cas de la station de traitement de Paspanga de l'ONEA au Burkina Faso. Mémoire de master de l'Institut International d'Ingénierie de l'Eau et de l'Environnement. $83 \mathrm{p}$.

Gaye S, Ouali A. 2014. L'influence de la concentration de bentonite industrielle sur la coagulation floculation décantation des eaux faiblement saumatres. Mémoire de master de l'Université Abou Bekr Belkaid, Algérie. $180 \mathrm{p}$.

Hernández De León HR. 2006. Supervision et diagnostic des procédés de production d'eau potable. Thèse de doctorat de l'Institut National des sciences Appliquées de Toulouse, France, 211 p.

Lamizana-Diallo MB, Kenfack S, MillogoRasolodimby J. 2008. Assessment of the physicochemical quality of a temporary river in Burkina Faso : the case of Massili in Kadiogo. Sud Sciences and Technologies, 23-28 p.

Moreau N. 2011. Manuel de l'Exploitation d'un Réseau AEP Gravitaire. Union des comités de l'eau Comores, p. 56. 
Mujawamariya M. 2012. Optimisation de la décantation primaire par ajout de produits chimiques - Jar-Tests. Mémoire de master de l'Université Laval, $90 \mathrm{p}$.

Nadjib DAA. 2017. Etude de l'alimentation en eau potable de la commune de tamalt a partir du barrage de tichi haff. Mémoire de Master de l'Université Abderrahmane MIRA de Bejaia, Algérie. 92 p.

NF EN ISO 5667-1. 2007. Qualité de l'eauéchantillonnage-partie 1 : lignes directrices pour la conception des programmes et des techniques d'échantillonnage (indice de classement : T90-511-1).

NF EN ISO 5667-3. 2004. Qualité de l'eauEchantillonnage- partie 3. Lignes directrices pour la conservation et la manipulation des échantillons d'eau (indice de classement : T90-513).

Njoyim EBT, Nchofua FB, Mofor NA, Djoko YT. 2016. Contrôle de la qualité des eaux domestiques dans le village Babessi au Nord-Ouest Cameroun. Int. J. Biol. Chem. Sci., 10(3) : 1382-1402. DOI : http://dx.doi.org/10.4314/ijbcs.v10i3.37

OMS (Organisation mondiale de la Santé). 2007. Combattre les maladies véhiculées par l'eau à la maison. Organisation Mondiale de la santé. 36 p. who.int/iris/bitstream/handle/10665/4375 6/9789242595222_fre.

OMS (Organisation Mondiale de la Santé). 2011. Directives de qualité pour l'eau de boisson. ISBN 978924154815 1, Genève, Suisse, $531 \quad \mathrm{p}$. https://www.who.int/water_sanitation_he alth/dwq/fr/

OMS (Organisation Mondiale de la Santé). 2012. Progrès en matière d'eau potable et d'assainissement, World Health Organization Geneva, 11-16 p.

OMS (Organisation Mondiale de la Santé). 2017. Les maladies liées à l'eau, https://www.who.int/fr/news-room/factsheets/detail/diarrhoeal-disease

Ouattara k. 2016. Evaluation de la matière organique des eaux de surface et son impact dans le processus de production d'eau potable. Mémoire de Master eau et assainissement 2ie Ouagadougou Burkina Faso, $65 \mathrm{p}$.

Ouattara Y, Guiguemde I, Diendere F, Diarra J, Bary A. 2012. Pollution des eaux dans le bassin du nakambe : cas du barrage de Ziga. Int. J. Biol. Chem. Sci., 6(6): 80348050. Doi http://dx.doi.org/10.4314/ijbcs.v6i6.47

Rodier J. 2005. L'analyse de l'eau naturelle, eaux résiduaires, eaux de mer (8ème Edition). Dunod technique : Paris, 10081043

Rodier J. 2009. L'Analyse de l'Eau Contrôle Et Interprétation (9ème édn). Dunod : Paris, p. 1579.

Sawadogo R, Guiguemde I, Diendere F, Diarra J, Bary A. 2012. Caractérisation physicochimique des eaux résiduaires de tannerie: cas de l'usine TAN ALIZ à Ouagadougou / Burkina Faso. Int. J. Biol. Chem. Sci., 6(6): 7087-7095. DOI : http://dx.doi.org/10.4314/ijbcs.v6i6.43.

Sommet de l'eau. 2013. L'eau est la clé du développement durable. Budapest, Hongrie.

https://news.un.org/fr/story/2013/10/276 072

Souha SAS. 2017. Dysfonctionnements biologiques dans les stations d' epuration a boues activees: cas de la station d'epuration de ain el hadjar. Mémoire de Master de l'Université de saïda - dr moulay tahar, $119 \mathrm{p}$.

Tapsoba FW, Kéré FD, Diarra J, Barry A, Sawadogo LH, Dianou D, Dicko MH. 2016. Etude de l'évolution des éléments précurseurs d'eutrophisation des eaux du Barrage $\mathrm{n}^{\circ} 3$ de Ouagadougou, Burkina Faso. Int. J. Biol. Chem. Sci., 10(2): 846859.

DOI: http://dx.doi.org/10.4314/ijbcs.v10i2.32

Tidiani BMI. 2017. Diagnostic du phénomène de remontée de boue dans le décanteur a lit de boue de la station de traitement d'eau potable de Goudel (NiameyNIGER). Mémoire de 2ie, Ouagadougou, 12-14 p. 